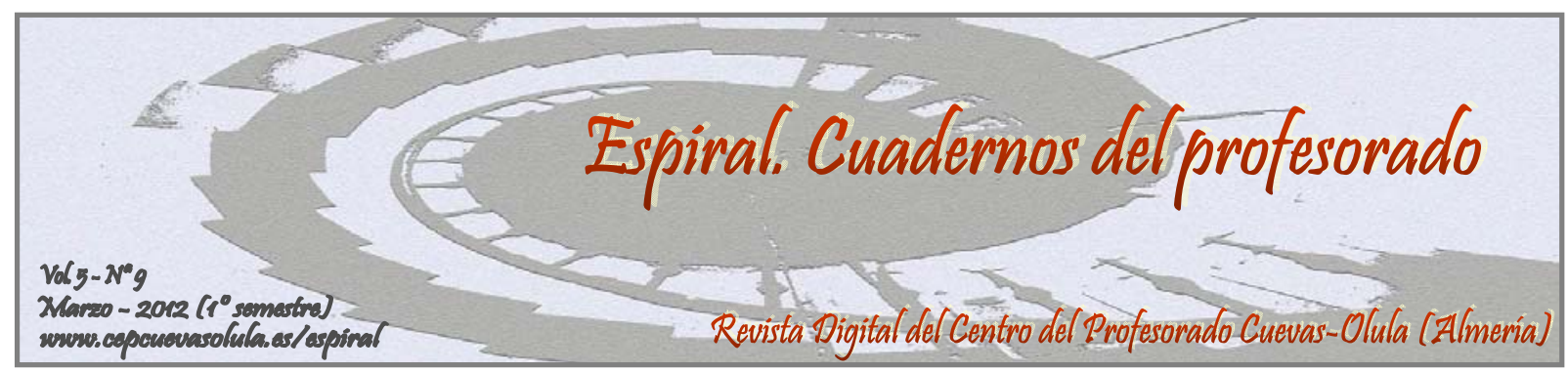

\title{
ANÁLISIS DE PRÁCTICAS Y VARIABLES FAVORECEDORAS DE PROCESOS INCLUSIVOS EN LOS CENTROS DE EDUCACIÓN SECUNDARIA OBLIGATORIA
}

\section{ANALYSIS OF VARIABLES AND PRACTICES TO FACILITATE INCLUSIVE PROCESSES IN THE CENTERS OF SECONDARY SCHOOL}

\author{
Vicente Jesús Llorent García ${ }^{(1)}$ y Rafael López Azuaga ${ }^{(1)}$
}

${ }^{(1)}$ Facultad de Ciencias de la Educación. Universidad de Córdoba, España

\begin{abstract}
RESUMEN: El objetivo principal de este trabajo fue diagnosticar las necesidades que presenta el profesorado de Educación Secundaria, de un centro educativo de Córdoba, para atender las necesidades educativas de su alumnado siguiendo los principios de la "educación inclusiva". Se trata de un estudio de caso, de análisis mixto, donde hemos encuestado a profesorado y alumnado, analizado recursos, realizado entrevistas etnográficas, etc. Se destaca que el centro educativo sigue fielmente la legislación educativa y que la participación de la comunidad educativa es muy limitada. Por otra parte, se han analizado las prácticas educativas con respecto a las siguientes variables: "Estrategias de Atención a la Diversidad”, "Participación de la comunidad educativa” y "Relaciones interpersonales y convivencia”. Esta investigación se ha nutrido de la información aportada por diferentes técnicas e instrumentos (entrevistas, cuestionarios y observación directa). Podemos comprobar que, aunque existe un interés por atender las necesidades educativas de todo el alumnado y por promover valores basados en la igualdad y la tolerancia (entre otros), aún hay aspectos a mejorar. Planteamos, por ello, una serie de propuestas de intervención.
\end{abstract}

Palabras clave: atención educativa a la diversidad, Relaciones interpersonales, Educación inclusiva, Educación Secundaria

ABSTRACT: We conducted a research with the primary aim of diagnosing the needs presented by the teachers of Secondary Education, an educational center of Cordoba, to meet the educational needs of their students on the principles of "inclusive education". This is a case study, combined analysis, where we surveyed teachers and students, discussed resources, conducted ethnographic interviews, etc. It is emphasized that the school closely follows the educational legislation and the education community participation is very limited. Moreover, we have analyzed the educational practices with respect to the following variables: "Strategies for Diversity", "Participation of the educational community" and "Relationships and coexistence." This research has drawn on information provided by different techniques and instruments (interviews, questionnaires and direct observation). We can see that although there is interest in meeting the educational needs of all students and to promote values based on equality and tolerance (among others), there are still areas for improvement. We propose, therefore, some intervention proposals.

Key words: educative attention to diversity, Interpersonal relationships, Inclusive education, Secondary Education. 
Llorent García, V.J., y López Azuaga, R. (2012). Análisis de las prácticas y variables favorecedoras de procesos inclusivos en los centros de educación secundaria obligatoria. Espiral. Cuadernos del Profesorado, 5(9), 3-15. Disponible en: http://www.cepcuevasolula.es/espiral.

Fecha de recepción: 13/10/2011

Fecha de aceptación: 10/01/2012
Enviar correspondencia a: vjllorent@uco.es

\section{1.- INTRODUCCIÓN}

En los últimos veinticinco años, han aumentado las medidas para atender la pluralidad de las aulas, incluyendo las necesidades educativas del alumnado que presenta "necesidades específicas de apoyo educativo" (NEAE). Se lleva a cabo el sistema de "integración educativa”, cuya idea principal consiste en que el alumnado con NEAE se encuentra matriculado en los centros ordinarios, pero en algunas horas o en la mayor parte o toda la jornada escolar éste se encuentre en aulas específicas. En dichas aulas especialistas con la debida formación específica se centran en el alumnado con dichas necesidades educativas.

Ante esta situación, existe un modelo alternativo, la "educación inclusiva”, en la cual se atiende a todo el alumnado partiendo de sus potencialidades y necesidades. Se parte de la idea de que todo el alumnado debe escolarizarse en el aula ordinaria, sin que nadie sea "excluido" a un aula específica. La misión de educar y atender las necesidades educativas es de todos y con todos. El equipo docente al completo debe trabajar cooperativamente en el proceso de enseñanza-aprendizaje y los especialistas, entre otras personas (familiares, voluntarios, trabajadores de otras instituciones, etc.), se encuentran dentro del aula ordinaria atendiendo a todo el alumnado, presenten o no NEAE (Muntaner, 2010,). Existen muchas variables que pueden influir en el desarrollo de una educación inclusiva, pero nosotros en este artículo nos hemos centrado en tres: "Estrategias de Atención a la Diversidad", "Participación de la comunidad educativa" y "Relaciones interpersonales y Convivencia”.

Con respecto a las estrategias de Atención a la Diversidad, podemos encontrar numerosas publicaciones científicas que recogen propuestas para favorecer la atención a las necesidades educativas. Si nos basamos en la Orden de 25 de julio de 2008 por la que se regula la atención a la diversidad del alumnado que cursa la educación básica en los centros docentes públicos de Andalucía, las principales medidas destacadas son las adaptaciones curriculares individualizadas y los programas de diversificación curricular, y en general otras medidas que recopilan los principios del sistema de integración educativa: Programas de Cualificación Profesional Inicial (PCPI), repetición de curso, programas de refuerzo de áreas instrumentales, agrupamientos flexibles, desdoblamiento de grupos en áreas instrumentales, etc.

Por otro lado, existen numerosos estudios que recogen medidas basadas en los principios de la educación inclusiva. Entre ellas, la principal medida consiste en diseñar prácticas que recojan los principios del "aprendizaje cooperativo". Esta propuesta educativa consiste en aprender en pequeños grupos de trabajo. Cada grupo está formado por miembros heterogéneos que trabajan en torno a un objetivo común y entre todos aportan ideas, se ayudan, se explican contenidos, fomentan el desarrollo de nuevas habilidades de los demás, investigan juntos, etc (Pujolàs, 2004). Evidentemente el uso de las TIC tiene mucho potencial en este sentido (Badia y García, 2006; Rodríguez, 2011).

Como ejemplo de experiencias, podemos mencionar a Lozano (2008), que aprovechando las posibilidades de los libros virtuales y de Internet, consiguió que alumnado con discapacidad intelectual pudiera desarrollar competencias lingüísticas y digitales gracias a la interacción con sus docentes y compañeros. De hecho, una de las alumnas discapacitadas fue capaz de exponer su trabajo y de asesorar a alumnado de grupos de edad inferior. Otra experiencia, dentro del ámbito no formal, es la llevada a cabo por la Asociación EQUA-UCA, donde un grupo formado por personas con discapacidad intelectual y personas sin discapacidad alguna, con asesoramiento previo, aprendiendo a autogestionarse y juntos organizan actividades culturales para la sociedad (López Azuaga, Rodríguez 
de Cossío, Sánchez Ruiz, et al, 2010). Está basado, por un lado, en los principios del constructivismo, en el cual partiendo de sus concepciones todos van investigando, trabajando y apoyándose los unos a los otros empleando diversos recursos didácticos, con las nuevas posibilidades del uso de las TIC (Salmerón, Rodríguez y Gutiérrez, 2010; Ramos y Martínez, 2011; Rodríguez, 2011) y evolucionando sus concepciones contando además con las orientaciones del profesorado (Navarrete, 1998). Por otro lado, recoge los principios del modelo interaccionista de Vigostky, donde los alumnos, desde una "zona de desarrollo próximo" (ZDP), van aprendido a desarrollar una serie de habilidades con ayuda de otras personas, para pasar posteriormente a una "zona de desarrollo real" (ZDR) en la cual son capaces de llevarlas a cabo de forma autónoma (Vigostky, 1979).

La participación de la comunidad educativa es fundamental para desarrollar una educación inclusiva. No solamente el profesorado debe ocuparse de la atención del alumnado, sino todas las familias deben participar en la medida de lo posible en el proceso de intervención de las necesidades educativas de sus hijos y en el diseño, desarrollo e innovación del currículum. Su participación en la organización y gestión de los centros educativos es fundamental, de forma que éstos puedan saber atender las necesidades de sus hijos, aplicable en entornos virtuales (Ramos y Martínez, 2011; Salmerón, Rodríguez y Gutiérrez, 2010). También el propio alumnado debe cooperar en estas tareas, al igual que otros miembros de diversas instituciones: voluntarios, pedagogos, psicólogos médicos, trabajadores sociales, educadores sociales, etc. Entre todos, pueden llevar a cabo "actuaciones de éxito" en los centros, como los grupos interactivos, tertulias literaria, bibliotecas tutorizadas, resolución de conflictos interactivamente, etc., y así desarrollar una "comunidad de aprendizaje”. En dichas comunidades, todos los miembros de la comunidad educativa, incluyendo de la propia sociedad, cooperan juntos en torno a la consecución de una serie de objetivos prioritarios, los "sueños", que se corresponden con la idea de cómo desearían que fuese su centro educativo "ideal". En dicho centro "ideal”, todas sus necesidades son atendidas y satisfechas (Aubert, García, Flecha, et al., 2008).

Finalmente, un clima de convivencia óptimo y adecuado permitirá el desarrollo de relaciones interpersonales positivas. Estas relaciones pueden favorecer el inicio y mantenimiento de una sociedad inclusiva, en la cual todos nos relacionemos y cooperemos todos juntos para desarrollar la sociedad, independientemente de nuestra caracterización.

El objetivo principal de nuestro estudio es diagnosticar cómo se están desarrollando las prácticas educativas en los centros educativos de forma que tengamos un punto de partida para desarrollar innovaciones centradas en la educación inclusiva, analizando las tres variables mencionadas.

\section{2.- MÉTODO}

Se ha desarrollado un estudio descriptivo con la finalidad de obtener suficiente información como para diagnosticar las necesidades del centro educativo con respecto a la educación inclusiva en las variables mencionadas. Hemos seleccionado un Instituto de Educación Secundaria de una localidad cordobesa que destacó por sus prácticas inclusivas en un estudio realizado por Arranz (2008). Hemos solicitado la colaboración de los 8 tutores/as de Educación Secundaria Obligatoria y de los 80 alumnos/as que se encuentran en el $2^{\circ}$ ciclo de Educación Secundaria. Obtuvimos una alta participación, pudimos recabar datos de 7 tutores/as y de 60 alumnos/as, lo cual lo consideramos representativo al superar ambos el $75 \%$ del total de la población. Los instrumentos que hemos utilizado para recoger información han sido los siguientes: Entrevistas etnográficas con el director y algunos alumnos/as del centro educativo, Informe con el análisis del contexto sociocultural y económico del centro educativo, Cuestionarios dirigidos al profesorado-tutor y al alumnado del $2^{\circ}$ ciclo de Educación Secundaria, Proyecto de Coeducación del centro educativo, Página Web del centro educativo (Actualidad del centro educativo, Diario de actividades realizadas y resultados de éstas, fotografías de las experiencias educativas, etc.) y el Estudio de Arranz (2008): "Proceso y procedimientos de evaluación inclusivos en contextos educativos".

Los cuestionarios fueron elaborados para cada colectivo, profesorado y alumnado, en base a las categorías que nos ayudasen a conocer las ya expuestas variables de nuestro estudio. A continuación detallamos las principales características de cada uno: 
1-Profesorado: Se han ordenado las preguntas por temática para ayudar a los participantes a activar sus "esquemas de conocimiento" y puedan relacionar toda la información que tienen acerca de su propia experiencia, además de ser útil para los investigadores a la hora de realizar los análisis de forma ordenada. En este estudio, nos hemos basado, en el caso del profesorado, en el apartado de la atención a la diversidad, con 13 preguntas, las cuales analizan cómo se desarrolla este ámbito, qué medidas se suelen emplear, cómo se desarrolla la colaboración entre el resto de miembros de la comunidad educativa (profesorado, alumnado, familias, otras instituciones), cómo se tiene en cuenta la diversidad cultural, qué mejoras propondría, etc, entre otras cuestiones.

2-Alumnado: Las diferentes preguntas del alumnado responden a las siguientes categorías: Cuestiones centradas en la concepción que mantienen sobre "diversidad" (2), el trabajo en el aula con estos compañeros y compañeras "diferentes” (4), las relaciones interpersonales entre el alumnado y los posibles casos de discriminación que se encuentran en su centro educativo (16), educación intercultural (6) y la participación del alumnado con o sin NEAE en el centro educativo (8).

Si bien debemos añadir que al final de los dos tipos de cuestionarios (profesorado y alumnado) se ha incluido un ítem sobre sugerencias o propuestas de mejora con respecto a la temática de las que les hemos encuestado.

La información aportada por cada una de las técnicas de recogida de datos ha sido clasificada en las siguientes categorías: "Estrategias de Atención a la Diversidad", "Participación de la comunidad educativa” y "Relaciones interpersonales y Convivencia”. Se realizará una triangulación de todos los datos que llevará a una serie de conclusiones que nos facilitará la elaboración de propuestas de mejora.

Con respecto a los cuestionarios, el único instrumento ad hoc de todas las técnicas utilizadas, entre las preguntas, hemos utilizado preguntas abiertas, preguntas con respuestas cerradas con opción de justificar su respuesta, escalas tipo Likert y preguntas de múltiple opción a raíz de aquellas alternativas que coincidan con el punto de vista del participante (marcar tantas como crea oportuno), siempre teniendo la opción de marcar la opción de “Otros” y concretar su elección.

Para las preguntas abiertas, se calculó el tanto por ciento de aparición de cada tipo de respuesta emitida, y se realizó un análisis en conjunto de todas ellas. Para los test y preguntas de opción múltiple, se calculó el tanto por ciento de cada una de las opciones. Finalmente, para las preguntas de escala Likert, se calculó la media $(M)$ y la desviación típica $(D T)$ en cada uno de los ítems, calculando además el tanto por ciento de sujetos que habían marcado la opción "No sabe/No contesta", por si se diese el caso de que fuese demasiado representativa, limitando el alcance de algunas conclusiones al respecto acerca de la significatividad de la media y desviación típica obtenida en dichos ítems. Hemos aplicado también la prueba de Kolmogorov-Smirnov $(K-S)$ para una muestra, la cual nos ayudará a ver si los resultados obtenidos en cada uno de los ítems de las escalas tipo Likert son fiables, para poder generalizar con respecto a la población objeto de estudio y a los valores esperados. Todos los datos obtenidos se triangularon, tanto cuantitativos como cualitativos. Hemos utilizado el programa informático SPSS 18 para realizar los análisis estadísticos.

Para asegurar la fiabilidad y validez del estudio, enviamos los cuestionarios y la propuesta de categorías del estudio a profesores con perfil similar al de profesor de instituto y especialistas, los cuales aportaron sugerencias con respecto a preguntas superfluas y expresiones que podrían dar lugar a ambigüedades. Entre todos hicimos un análisis en profundidad sobre el lenguaje empleado en los cuestionarios, para evitar que pudiese ser demasiado complejo o ambiguo. Siguiendo con los cuestionarios, también contamos con la participación de una muestra de docentes y alumnos con los cuales realizamos una "experiencia piloto" para comprobar que los cuestionarios permitían recoger adecuadamente la información que deseábamos, a raíz de las respuestas emitidas por los participantes. Se le añadió una pregunta más en las cuales les consultábamos si añadirían o modificarían algo del cuestionario.

Con respecto a las técnicas estadísticas utilizadas, se aplicó el Alpha de Cronbach para asegurar la fiabilidad del estudio a cada una de las escalas Likert. La escala utilizada para el profesorado da lugar a un Alpha de Cronbach $=.57$, siendo algo bajo pero puede justificarse debido al bajo número de docentes participantes en el estudio al ser una población pequeña. La prueba $K-S$, a un 
nivel de confianza del 95\%, nos aporta en cada uno de los ítems resultados superiores a $\mathrm{p}=.05$ en la significación bilateral, tal vez por ser una población demasiado pequeña. En el caso de la escala utilizada en el cuestionario dirigido al alumnado, hemos obtenido un Alpha de Cronbach $=.78$, lo cual nos indica que la fiabilidad de dicha escala es muy positiva. La mayoría de los ítems, una vez realizada la prueba $K-S$ a un nivel de confianza del $95 \%$, nos ha dado un resultado inferior a $\mathrm{p}=.05$ en la significación bilateral, por lo que prácticamente podemos generalizar los resultados a la población.

\section{3.-RESULTADOS E INTERPRETACIÓN DE LOS RESULTADOS}

\section{Estrategias de Atención a la Diversidad}

Un 85.7\% afirma que el alumnado con NEAE se encuentra escolarizado en el aula ordinaria solo que durante algunas horas sale del aula ordinaria para recibir pequeños apoyos. Entre las estrategias más frecuentes, un $85.7 \%$ afirma que los alumnos y alumnas con mayores dificultades se encuentran en un programa de diversificación curricular y un $71.4 \%$ que estos alumnos y alumnas tienen una adaptación curricular individualizada (ACI). Las medidas más frecuentes son aquellas relacionadas con la integración educativa, siguiendo la actual Orden de 25 de julio de 2008 por la que se regula la atención a la diversidad del alumnado que cursa la educación básica en los centros docentes públicos de Andalucía, por lo que no se destacan medidas inclusivas dentro de las aulas.

El $85.7 \%$ del profesorado requiere una mayor disponibilidad de recursos humanos y un $57.1 \%$ de recursos materiales, lo cual confirma que el profesorado se siente incapacitado dada su escasa formación inicial y por ello requiere la presencia de más profesionales en el aula. El estudio de Arranz (2008) confirma que los centros de Secundaria participantes del estudio no están demasiado satisfechos con los recursos y servicios de apoyo disponibles en el centro y en el entorno.

El 100\% del profesorado-tutor exige más recursos humanos con especialidades específicas de forma que pueda ser una ayuda cuando se encuentren con ciertas necesidades en el aula. Un $71.4 \%$ exige que la Administración debería reducir las tareas burocráticas y administrativas para favorecer tener mayor disponibilidad para centrarse en planificar las medidas de intervención y en el proceso de enseñanza-aprendizaje de sus alumnos. También, coincidiendo con el anterior análisis, requieren por parte de ella una mayor cantidad de recursos materiales para atender las necesidades del centro educativo (71.4\%) y para desarrollar metodologías adaptadas a las necesidades educativas (57.1\%). Aún así, en general se destacan todas las ideas presentadas en la pregunta, puesto que un $42.9 \%$ considera que sería una gran ayuda aportarles mayor financiación económica para así ellos administrar dichos recursos económicos de forma que atiendan a sus necesidades más prioritarias.

Con respecto a la información aportada por el alumnado, tenemos los siguientes resultados: "Realizamos actividades en grupo" ( $M=2.88, D T=.91)$ y "En los trabajos en grupo, cuando el profesor organiza los grupos, me sientan con alumnos de diferentes culturas, del sexo opuesto, homosexuales, extranjeros, feos o simplemente que no son amigos míos" $(M=2.51, D T=1.08)$. Se contrasta con la información obtenida por los materiales recopilados del centro (diario de actividades, proyecto de coeducación, etc.) y por lo aportado por el profesorado con respecto al uso de estrategias de trabajo en grupo en las aulas (fundamental para la Atención a la Diversidad si se plantea de forma adecuada), aunque el profesorado debe preocuparse por formar grupos más heterogéneos para favorecer que el aprendizaje cooperativo pueda ayudar a desarrollar nuevos conocimientos y habilidades a cada uno de los alumnos y alumnas, ayudándoles a superar dificultades e incluso permitiendo que los alumnos afiancen sus conocimientos y habilidades cuando se las han transmitido a otros compañeros/as. De esta manera, se favorecería una educación más inclusiva.

\section{Participación de la comunidad educativa}

El 100\% del profesorado-tutor requiere la cooperación del Departamento de Orientación para recibir ayuda sobre las necesidades educativas del alumnado, lo que demuestra que el profesorado, independientemente de lo que piense acerca de que es misión suya atender al alumnado con NEAE o no, es 
consciente de las exigencias que le demandan en la actualidad y que debe saber adaptarse a ellas, y dada su cuestionada formación inicial (más adelante analizaremos este dato), requiere ayuda, y gracias al apoyo y la interacción continua entre ambos, el profesorado adquirirá nuevas habilidades para atender las necesidades educativas, fundamental en un sistema de inclusión. Por su parte, otro dato positivo es que un $71.4 \%$ del profesorado afirma coordinarse con dicho departamento para diseñar actividades cooperativas, esencial para alcanzar una inclusión educativa (aunque habría que analizar cómo se desarrollan dichas actividades cooperativas). Cabe destacar que el asesoramiento es nulo en otras estrategias, como el asesoramiento en el diseño, desarrollo e innovación del currículum, la ayuda del especialista dentro del aula educativa (confirmando lo establecido de que se sigue lo dictado por la normativa, influida por el sistema de integración educativa) o el desarrollo de una "comunidad de aprendizaje”. Los materiales recopilados confirman la intervención del Departamento de Orientación en el desarrollo de proyectos educativos, como elaborar materiales para las actividades del proyecto de Coeducación (exposiciones, material didáctico, confección de carteles, sesiones para la hora de tutoría lectiva, etc.). Cabe destacar que en el proyecto de Coeducación todos los departamentos didácticos elaboran actividades para promover la igualdad de género y el rechazo a las conductas de discriminación y a la violencia de género: Lectura de textos en otros idiomas sobre género, análisis de hechos y datos estadísticos sobre la violencia de género, mujeres palestinas y derechos humanos, el papel de la mujer en el avance del conocimiento matemático, la situación de la violencia de género en Córdoba, etc.

Con respecto a la participación de las familias, un $85.7 \%$ afirma que las familias asisten a las reuniones de padres y madres que se convocan a principios de curso y a las tutorías, y este resultado es contrastado medianamente por la información aportada por el alumnado $(M=2.71, D T=1.08)^{1}$. Un $57.1 \%$ ayuda a sus hijos/as con los estudios y aportan datos sobre el contexto sociofamiliar, siendo esto último fundamental para favorecer la evaluación psicopedagógica de los alumnos ${ }^{2}$. Las dos primeras medidas de participación son las principales medidas de interacción entre profesorado y familia establecidos en la Orden de 27 de julio de 2006 por la que se regulan determinados aspectos referidos al Plan de Orientación y Acción Tutorial en los Institutos de Educación Secundaria. Es nula su participación en actividades extraescolares; diseño, desarrollo e innovación del currículum, asistencia y participación en reuniones del Consejo Escolar, desarrollo de un "Grupo de Ayuda Entre Profesorado" (GAEP) y de una comunidad de aprendizaje, principales medidas para que el centro educativo pueda ser capaz de atender las necesidades e intereses de las familias además de que éstas puedan cooperar en el proceso educativo de sus hijos/as, sobre todo en atender sus necesidades educativas. La información aportada por el alumnado confirma la baja participación de las familias en actividades y festividades organizadas por el centro educativo $(M=1.63, D T=.85)^{3}$. El diario de actividades consultado confirma que éstas no tienen un papel significativo, ya que ni se les menciona como participantes en dichas actividades. Con respecto al estudio de Arranz (2008), el profesorado también consideraba que la participación de las familias no le parecía suficiente, considerando que habría que buscar alternativas que aumentasen y mejorasen la participación.

Con respecto al alumnado, en este centro educativo es el miembro que más participa en el centro educativo. Un 57.1\% destaca su participación en las siguientes actividades: Participación en el consenso de normas y valores y ayudan durante el desarrollo de actividades extraescolares. Tenemos como referencia los resultados de diferentes actividades en las cuales el alumnado ha tenido un rol activo, y su implicación ha sido fundamental para favorecer su participación: Gymkhana coeducativa de las tareas domésticas, representaciones de obras teatrales, actuaciones de flamenco, celebración de la fiesta de Halloween, preparación de exposiciones relacionadas con la violencia de género, etc. Los delegados y delegadas, por su parte, además de participar en estas tareas, asisten a la junta de delegados

\footnotetext{
${ }^{1}$ Debemos mencionar aquí que en esta escala solamente pudo añadirse 4 ítems en la aplicación por el hecho de haber incluido el ítem "No sabe/No contesta". Tendremos en cuenta las siguientes descripciones verbales: "Nada de acuerdo", “A medias", "De acuerdo", "Muy de acuerdo".

${ }^{2}$ Ver la Orden de 19 de septiembre de 2002, por la que se regula la realización de la evaluación psicopedagógica y el dictamen de escolarización.

${ }^{3}$ Escala de 1 a 4 con descriptores verbales que ayudaban a realizar el análisis.
} 
(85.7\%) y se entrevistan con los docentes para comentarles cualquier asunto relacionado con su grupoclase (57.1\%). Como se refleja, coincide con la normativa vigente: El Decreto 327/2010, de 13 de julio por el que se aprueba el Reglamento Orgánico de los Institutos de Educación Secundaria, el cual señala estas acciones como las principales tareas de los delegados, entre otras posibles que puedan establecerse en cada centro educativo. También se les consultó al alumnado acerca de su participación en el centro educativo, y se confirman los resultados en un término medio : "Colaboro con mis profesores en la preparación de actividades" $(M=2.56, D T=.86)$, "Realizo sugerencias para mejorar el funcionamiento y la dinámica de las clases" ( $M=2.26, D T=.89)$, "Realizo sugerencias para mejorar el ambiente, dinámica y convivencia de mi instituto" $(M=2.26, D T=.86)$, "Suelo ayudar a los alumnos que tienen más dificultades en clase" ( $M=2.31, D T=.90)$. También se confirmó la asistencia de los delegados y delegadas a la Junta de Delegados a partir de los alumnos: "Existe una Junta de Delegados" ( $M=3.19, D T=.89)$, aunque existe un $20 \%$ de alumnos y alumnas que han respondido "No sabe/No contesta". ¿Quiere decir que no se encuentran informados cuando el delegado asiste a una junta, y no suele comentarles aquello que se ha debatido en dicha junta? Es una pregunta que habría que analizar más detenidamente.

Con respecto a la coordinación del profesorado con sus compañeros/as docentes, el 100\% afirma compartir sus dudas, problemas, discrepancias, etc., un 85.7\% cooperan aportándose datos para apoyar el diagnóstico y evaluación del alumnado, un 71.4\% coopera para atender a las necesidades educativas del alumnado y en su intervención, y un 57.1\% en diseñar planes de actuación ante necesidades encontradas. Nos parece muy positivo este nivel de cooperación para favorecer la atención de las necesidades educativas de una forma satisfactoria y apoyarse entre ellos de forma que enriquezcan sus conocimientos, aunque no especificaron que desarrollasen una "comunidad de aprendizaje". Gracias a la cooperación entre profesorado y a la participación del alumnado, se aproximan a la idea de "comunidad”, aunque aún es necesario seguir evolucionando este aspecto. Aún así, suele entrar dentro de las pautas concretadas en la Orden de 27 de julio de 2006 por la que se regulan determinados aspectos referidos al Plan de Orientación y Acción Tutorial en los Institutos de Educación Secundaria. El estudio de Arranz (2008), sin embargo, considera que se deberían mejorar las estrategias y condiciones para desarrollar el trabajo cooperativo en la programación del aula, al no haber sido muy valorada por el profesorado ( $M=2.9, D T$ desconocida).

Con respecto a la existencia de instituciones de la comunidad que cooperen con el centro educativo, un $85.7 \%$ destaca la cooperación de organizaciones no gubernamentales y de asociaciones en el desarrollo de actividades en el centro educativo y un $71.4 \%$ de centros sanitarios, como las principales instituciones más mencionadas por el profesorado. Sin embargo, el alumnado contradice estos resultados, al valorar los siguientes ítems de la siguiente manera": "Soy consciente de que vienen especialistas de otras instituciones de la comunidad a colaborar con mi instituto" ( $M=2.95, D T=0.79$, $\mathrm{NS} / \mathrm{NC}=28.3 \%$ ), "Mi instituto se encuentra dentro de un programa educativo en el que colabora con otros centros educativos" $(M=2.81, D T=0.92, \mathrm{NS} / \mathrm{NC}=40 \%)$. Puede ser debido a que el alumnado no está informado acerca del papel que tienen profesionales de otras instituciones en posibles proyectos educativos que se lleven a cabo. Puede que tengan un papel más de asesoramiento hacia el Departamento de Orientación y al profesorado en lugar de una intervención más directa con el alumnado. De todas formas, las evidencias que hemos encontrado en el diario de actividades, incluyendo fotografías que prueban que dichas actividades se realizaron, contradicen estos resultados. Entre las actividades, en las cuales cooperaron la Universidad de Córdoba, asociaciones o especialistas de diversas instituciones: "La ciencia para todos", representaciones de Romances ciegos por parte de una compañía teatral, taller de escritura creativa en la Biblioteca municipal, Actividad "Biodiversos" organizada por el Ayuntamiento, visita a la empresa COVAP, visita a la fábrica de muebles de Hiansa S.A, Parque de las ciencias de Granada, etc. Puede que no hayan entendido bien lo que se les preguntaba o interpretan "participación” con presencia continua en el centro educativo, cuando este tipo de tareas suelen ser más puntuales. Con respecto a la cooperación con otros centros educativos, aquí sí coincide con el pro-

\footnotetext{
${ }^{4}$ Escala de 1 a 4 con descriptores verbales que ayudaban a realizar el análisis.

${ }^{5}$ Escala de 1 a 4 con descriptores verbales que ayudaban a realizar el análisis.
} 
fesorado a la hora de manifestar su confusión, porque solamente el 14.3\% manifiesta cooperar con otros centros educativos. Como mucho, sí hemos encontrado que el centro educativo participa en competiciones con otros centros educativos, siendo éstas organizadas por el CEP, por ejemplo la competición “Sex-Joven”, sobre violencia de género.

Finalmente, destacamos que un 57.1\% del profesorado considera que deberían intentar aumentarse en el centro educativo la colaboración del alumnado y de las familias para favorecer la Atención a la Diversidad.

\section{Relaciones interpersonales y convivencia}

El profesorado destaca que el alumnado se sensibiliza positivamente con el alumnado que presenta discapacidad $^{6}(M=3, D T=0)^{7}$ y que no suelen frecuentar las actitudes de discriminación hacia alumnos/as por género $(M=1.43, D T=.54)$, raza o etnia $(M=1.43, D T=.54)$ y por orientación sexual $(M=1.86, D T=1.22)$. Tal vez podríamos haber incluido un ítem en el cual se les preguntase sobre la discriminación hacia alumnos/as con altas capacidades intelectuales.

Centrándonos en el alumnado, hemos analizado el concepto de "diferente" que presentan como una manera de ver cómo influye esto en su manera de relacionarse con las personas. Ha habido mucha variedad de respuestas, pero las más frecuentes han sido la de considerar como "diferentes" a aquellos compañeros/as que presentan características diferentes a las que están acostumbrados a ver (15.9\%), aquellos alumnos que tienen un "comportamiento extraño" (14.5\%) y aquellos alumnos/as que no se relacionan socialmente con los demás (17.4\%). Los dos primeros pueden combinarse al estar relacionados en su significado interior (por ello, formarían un 30.4\%), y tiene su origen en que muchos hemos estado siempre acostumbrados a situarnos a nosotros mismos como el prototipo ejemplar de persona, es decir, que siempre todas las personas de nuestra edad y entorno deben comportarse de la misma manera para ser considerados como "normales". También ayuda a justificar la primera de las respuestas, puesto que siempre que vemos que alguien no se comporta de la manera ideal acorde a nuestras preferencias, filosofía de la vida, escala de valores, moralidad, etc., pues se le etiqueta como “diferente”. Esto también se justifica comprobando que el 13\% del alumnado ha señalado que consideran "diferentes" a alumnos/as con diferente personalidad, gustos y forma de pensar. La principal causa que incita a que el alumnado construya dichas concepciones y sistemas de "categorización" son sus experiencias personales vividas en su contacto con el entorno (Navarrete, 1998). Con respecto a los alumnos/as que no se relacionan socialmente con otros compañeros, esto se justifica en base a que, siguiendo a Guil (2004), los seres humanos tenemos necesidad de relacionarnos socialmente y de compartir nuestros sentimientos y emociones. Si vemos que una persona se niega a realizar esta acción por diversos motivos (no siempre va a ser porque el sujeto tenga un trastorno generalizado del desarrollo), y no lleva a cabo actividades compartidas con sus compañeros/as y ni se preocupa por ellos/as y se "encierra", termina resultándoles extraño. Esto hace que se relacione con las otras respuestas. El hecho de considerarle "diferente" se refuerza por el hecho de no conocer de manera significativa a esa persona, por lo que, al resultar "semi-desconocida", sigue siendo un "extraño" para nosotros aunque lleve tiempo en nuestra aula. Es a través de la comunicación como las personas compartimos conocimientos, sentimientos, emociones y nos desarrollamos, por eso se considera como una acción vital e imprescindible del ser humano para su desarrollo personal y construir su identidad y subjetividad (Díaz Gómez y González Rey, 2005). Si alguien rechaza realizar esa acción a la vez que necesidad vital, se considera "raro" por rechazar las leyes de la naturaleza. En cuanto al resto de respuestas, se ajustan a los análisis realizados por Sánchez Palomino y Lázaro (2011), siendo estas definiciones de "diferente" basadas en la discriminación, en la marginación o en intentar "curarle" o "corregirle" para que deje de ser "diferente". Este tipo de reacciones son alimentadas por la ignorancia, el mito, la superstición y sobre todo el miedo a lo desconocido. Aquello que no se asemeja dentro de sus concepciones sobre

\footnotetext{
${ }^{6}$ Escala de 1 a 4 con descriptores verbales que ayudaban a realizar el análisis.

${ }^{7}$ Esta desviación típica puede parecer muy extraña, pero al ser una población tan pequeña ( $\mathrm{n}=7$, sobre 8 tutores), no parece tan dificultoso que todos hayan marcado el valor 3 al valorar este ítem.
} 
"normalidad” en cada una de las categorías de la vida (intelecto, personalidad, gustos, etc), será considerado como "diferente".

Acerca de la posible discriminación o no que puedan sufrir estos alumnos/as, nos resulta positivo ver que solamente un $36.7 \%$ afirma que sí sufren discriminación, aunque no es todo lo bajo que desearíamos. En la escala Likert, se confirma un término con una desviación típica superior a 1 con respecto a la presencia de casos de discriminación en las aulas: "Hay alumnos que se meten con otros compañeros" $(M=2.49, D T=1.01)^{8}$. Debido a que existen muchas respuestas diversas a la hora de valorar la discriminación o no que sufren los compañeros/as, realizaremos un análisis en conjunto de todas ellas. Cabe destacar que un $20 \%$ ha dejado la pregunta de "justificación” en blanco. En cuanto al resto, un 28.3\% afirma respetar a sus compañeros/as, sean "diferentes" o no, por diversas razones: "Nos conocemos desde hace muchos años y por ello somos amigos", "Todos somos personas y por ello no nos insultamos los unos a los otros", "Normalmente solemos comunicarnos con los compañeros "diferentes", "No discriminamos a nadie porque todos somos normales", "Normalmente solemos respetar a todos los compañeros, independientemente de cómo sean". Se encuentran aquí reflejados valores relacionados con el respeto y la tolerancia, puesto que no consideran que existan motivos para discriminar de forma justificada a una persona. Sin embargo, un $40.1 \%$ de los que han respondido a la pregunta exponen que sus compañeros son discriminados por su caracterización, algunas de ellas por el hecho de presentar un caracterización "diferente" a las que están habituados a ver y en otros casos por presentar una caracterización que la justifican como "perjudicial" para ellos o por otras razones: "El sujeto presenta mal olor", "No discriminamos a nadie, pero hay personas con las que no nos interesa relacionarnos", "Me dan miedo", "No cuentan con él para jugar", "Presenta un comportamiento inadecuado para nosotros", "Tiene dificultades con los estudios", "Les insultan con frecuencia”. Aunque podríamos haber intentando profundizar más en las causas, podemos ver que existen casos de discriminación en las aulas y una de las variables que influyen es la caracterización del sujeto, a la cual consideran que, por ser como es, no debe ser tratado como a un "igual" o al menos como le gustaría a una misma persona que le tratasen. Ven esa diferencia como algo inadecuado, relacionado con las justificaciones de ignorancia y miedo que sienten estos alumnos cuando se encuentran algo diferente, con respecto a los análisis de Sánchez Palomino y Lázaro (2011). Por otra parte, un 8.3\% destaca que son los propios sujetos los que se aíslan y no se relacionan de forma adecuada, lo que conlleva a que sean "discriminados": "El propio sujeto se aísla de los demás", "Se mete con todo el mun$d o$ ". Esto se contrasta con el resultado anterior obtenido de que se considera "diferente" a aquellas personas que no se relacionan socialmente con los demás y que presentan unas habilidades sociales y actitudes que deben ser evolucionados de forma positiva para favorecer las relaciones interpersonales. ¿Y qué opina el profesorado sobre la discriminación en las aulas? El porcentaje de los que afirman que se produce discriminación en las aulas también es bajo (28.6\%), aunque se destacan las siguientes razones, las cuales contradicen en cierta medida lo aportado por el alumnado: "Alumno que procede de otro centro educativo", "Discriminación por género". ¿Por qué sucede esto? Siendo ambas muestras representativas, nos lleva a la conclusión de que cada uno interpreta los sucesos desde su propia perspectiva a través de la información de la que dispone. Puede que vean que se discrimina a un alumno/a que viene de otro centro educativo pero, ¿es esa realmente la razón por la que es discriminado? ¿No presenta este sujeto alguna otra característica que hace que sea discriminado, como las que expuso el alumnado? Tal vez esta sea la cuestión, y podría profundizarse a través de entrevistas y observación a nivel etnográfico.

Como dato llamativo, un 73.3\% del alumnado reconoce que no se suelen juntar con esos compañeros/as “diferentes” para relacionarse socialmente. Un 23.3\% afirma que tienen sus propios amigos y amigas para relacionarse y que por ello no tienen necesidad de relacionarse con esos compañeros/as. Haciendo un análisis general de la diversidad de respuestas obtenidas, podemos llegar a la conclusión de que el alumnado que no se relaciona con estos compañeros/as "diferentes" es debido a que presentan una caracterización que se desconecta con su prototipo de "normal”, o simplemente que cuando no coincide con la suya piensan que una relación social con ellos no va a funcionar. Un $23.5 \%$ aporta co-

\footnotetext{
${ }^{8}$ La escala era de 1 a 4, por las razones antes expuestas y que ayudaban a realizar el análisis.
} 
mentarios relacionados con este hecho: "Me dan miedo", "Sus temas de conversación no me interesan", "No realizan las mismas actividades que yo en el recreo", "No me relaciono con ellos porque tienen gustos diferentes a los míos", "Solamente me relaciono con ellos cuando me interesan por algún motivo", "No le veo capacitado como para entenderme", "Yo tendría dificultades para entenderlos", "Ellos tendrían dificultades para entendernos", etc. De nuevo tenemos aquí un ejemplo de reacción social ante la diferencia, en este caso la marginación como una forma de responder ante la diferencia, basándose en la ignorancia y el miedo al contactar con algo "innovador" para ellos/as. Este tipo de conductas puede promover la tendencia a generar estereotipos y prejuicios acerca de las personas que presenten una caracterización específica, estableciendo generalizaciones hacia todas aquellas personas que presenten características similares (Guil, 2004). Finalmente, un $11.7 \%$ afirma que esos compañeros/as se relacionan con otras personas.

Analizando la escala Likert $^{9}$, destacamos como muy positivos los siguientes resultados: "Hay compañeros que prefieren reunirse en el recreo con sus amigos españoles que con amigos de otros países" ( $M=1.73, D T=.89)$, "No tengo problemas en ver a amigos míos hacer tradiciones populares de sus países de origen o de otros países" $(M=3.41, D T=.86)$, "No tengo ningún inconveniente en sentarme con compañeros del sexo opuesto" ( $M=3.86, D T=.48)$, "No tengo ningún inconveniente en sentarme con compañeros que presenten discapacidad" ( $M=3.45, D T=.76)$ y "No tengo ningún inconveniente en sentarme con compañeros de diferentes culturas" $(M=3.52, D T=.81)$. En general, se demuestra que el alumnado en general no tiene inconvenientes en relacionarse con compañeros/as por razones de etnia, discapacidad y género, aunque existe mayor heterogeneidad en las opiniones sobre el ítem "No tengo ningún inconveniente en sentarme con compañeros homosexuales, bisexuales o transexuales" $(M=3.06, D T=1.08)$, tanto por su desviación típica como porque un $10 \%$ del alumnado ha respondido "No sabe/No contesta".

En general, se tienen en cuenta otras características a la hora de decidir discriminar a alguien, relacionadas con comportamientos que no están habituados a ver en su contexto. No consideran extraños a compañeros/as que presenten diversidad por razones de género, etnia o discapacidad porque están habituados a raíz de que se ha favorecido la escolarización de alumnado con dichas características en los centros educativos ordinarios. De todas formas, con respecto al primer ítem señalado, existe un $25 \%$ que ha respondido "No sabe/No contesta". Quizás pueda deberse al hecho de que no existan demasiados alumnos/as inmigrantes en el centro educativo en relación con lo aportado por el profesorado, y por ello se basan más en sus experiencias personales. Confirmamos la validez de este dato al contrastarlo con la información aportada por la Delegación Provincial, en el cual solamente un 0.12\% del alumnado inmigrante de la localidad de Córdoba se encuentra escolarizado en este centro educativo. No obstante, para una próxima vez podríamos haberles preguntado si tenían compañeros inmigrantes o de minorías culturales en las aulas.

En las técnicas cualitativas, hemos podido analizar en las fotografías la cooperación que existe entre los alumnos en actividades cooperativas y una actitud positiva durante la realización de actividades extraescolares o eventos especiales en el centro educativo. Trabajan juntos tanto chicos como chicas mezclados en grupos heterogéneos. Para mejorar la convivencia, en el centro educativo se lleva a cabo el Proyecto de Coeducación, que promueve las relaciones de igualdad de género y el rechazo a actitudes de discriminación hacia las mujeres, haciendo especial hincapié en la violencia de género. También se analiza el papel de la mujer en la sociedad y a lo largo de la historia, como una forma de ver cómo el género no influye a la hora de que una persona pueda aportarnos sus conocimientos y habilidades para mejorar la sociedad, eliminándose todo tipo de estereotipos sobre las mujeres, por ejemplo, demostrando que también hay hombres realizando trabajos que históricamente se les habían atribuido a las mujeres y viceversa. Entre las actividades, se destacan: Charlas, visionado de películas, análisis de canciones, lecturas sobre la situación de la mujer en Francia y los países francófonos, Gymkhana de tareas domésticas, proyectos educativos sobre violencia de género, exposiciones en las que se recogen las aportaciones de las mujeres en diversos campos del saber, historia de la lucha de la mujer por sus derechos, exposiciones de fotografías de los docentes del centro realizando tareas do-

\footnotetext{
${ }^{9}$ En este caso, también la escala fue de 1 a 4 con descriptores verbales, por las mismas razones.
} 
mésticas, mesa redonda con mujeres desempeñando puestos que tradicionalmente eran ocupados por hombres, etc. En general, se fomentan unos valores inclusivos que promueven el respeto entre los hombres y las mujeres y el rechazo hacia actitudes de discriminación. Hemos podido encontrar numerosas fotografías y murales sobre las experiencias.

\section{4.- DISCUSIÓN, CONCLUSIONES Y SUGERENCIAS PARA LA PRÁCTICA DOCENTE}

Podemos comprobar que se intenta crear un clima de convivencia y de inclusión en el centro educativo. El proyecto de Coeducación, entre otras tareas, parece dar sus frutos en el alumnado a raíz de sus actitudes hacia la diversidad, pero aún existen casos de discriminación que deben ser tratados, además de favorecer al alumnado con más dificultades para relacionarse puedan mantener unas relaciones interpersonales más positivas con sus compañeros/as con el objeto de favorecer su desarrollo y aprendizaje a través de la interacción interpersonal. Si analizamos con mayor detenimiento cada variable estudiada, una razón posible por la que el profesorado no emplee estrategias didácticas para la Atención a la Diversidad alternativas a las planteadas por la integración educativa sea porque necesiten más información sobre su desarrollo. Puede tomarse como referencia los estudios de la Agencia Europea para el Desarrollo de la Educación Especial (2003); Moliner, Sales, Fernández et al (2008), Colmenero (2006), Moliner y Moliner (2007), los cuales confirmaban que el profesorado de Educación Secundaria no presentaba una formación en estrategias para la educación inclusiva y que incluso desconocían el concepto de "inclusión educativa". El sistema de categorización que emplea el alumnado para definir a una persona como "diferente” se asemeja al planteado por Sánchez Palomino y Lázaro (2011). Por su parte, la limitada participación de la comunidad educativa en los centros educativos, centrándose sobre todo en lo establecido por la legislación educativa vigente, confirma la necesidad de darle más voz a todos y todas para que así todos puedan aprender de todos y transmitir sus preocupaciones, de forma que puedan ser mejor atendidas y conseguir una escuela más inclusiva (Susinos y RodríguezHoyos, 2011).

¿Qué prácticas podríamos desarrollar en las aulas? Para desarrollar actitudes y valores positivos relacionados con la tolerancia, la solidaridad, la empatía, la ayuda, etc, podríamos desarrollar tareas como las siguientes: Dilemas morales, dinámicas de grupo, proyectos de investigación, talleres interculturales, desarrollo de murales y exposiciones con la correspondiente "evaluación entre iguales", juegos de roles, foros de discusión sobre el contenido de diversos recursos (vídeos, lecturas juveniles, relatos, etc), resolución de conflictos en el aula... (Araque, 2009).

También sería muy útil promocionar en las aulas las políticas de la Unión Europea sobre igualdad de trato y no discriminación a través de unidades didácticas, desarrollar programas de habilidades sociales, desarrollar la inteligencia emocional, trabajar el tema del acoso escolar o "bullying" en las aulas (incluyendo el "ciberbullyng”)... (Ma, 2008). Como sugerencia personal nuestra, podríamos crear una red social, propia del centro educativo, en la plataforma NING para favorecer que todo el alumnado se relacione entre ellos/as, conozcan a nuevos compañeros/as y aquellos/as que sean más tímidos se les incite a relacionarse con los demás, empezando por escrito y poco a poco será capaz de dirigirse a los demás oralmente.

La cooperación del profesorado con la comunidad educativa es diversa, destacando la alta implicación del alumnado. Principalmente el punto débil de la participación se encuentra en la familia, cuya implicación se basa prácticamente en la asistencia a las reuniones de padres que se convocan a principios de curso y la asistencia a tutorías. Resulta fundamental buscar nuevas estrategias que favorezcan su participación. Nosotros proponemos aprovechar las TIC para mantener una comunicación online de forma que los padres puedan comunicarse con los tutores, estar al tanto del calendario escolar, seguir el proceso educativo de sus hijos y las tareas que deben realizar, entre otras tareas (Ramos y Martínez, 2011; Salmerón, Rodríguez y Gutiérrez, 2010); realizar encuestas de satisfacción a los padres, intentar buscarle un rol a los padres en las actividades que se realicen en el centro educativo (charlas, ayudar al alumnado, controlar el comportamiento del alumnado...), realizar más talleres formativos en el centro educativo para que puedan saber cooperar con el profesorado en el proceso educativo de sus hijos/as... (Susinos, Rodríguez-Hoyos, 2011). 
Con respecto al Departamento de Orientación, dada la desorientación del profesorado acerca de estrategias que recogen los principios de educación inclusiva, encontramos la necesidad de asesorar más al profesorado para promover más tareas cooperativas dentro del aula, favorecer que el alumnado con mayores dificultades y mayores potencialidades se encuentre en grupos de trabajo heterogéneos para aprender nuevos conocimientos y habilidades gracias al contacto con sus compañeros/as a la vez que éstos puedan aprender de él y asesorarles acerca de la construcción de una "comunidad de aprendizaje” en el centro educativo, en el cual las familias, voluntarios, especialistas de otras instituciones accedan al centro educativo y cooperen en el proceso educativo de forma que gracias al trabajo de todos/as logren alcanzar una serie de objetivos y "aspiraciones" para conseguir que el centro educativo sea el “ideal” para todos (Aguado y otros, 2007; Martínez y Álvarez, 2005).

El Departamento de Orientación también podría insistir más, en cooperación con todos los miembros del centro, en el desarrollo efectivo del diagnóstico de los recursos humanos y materiales que se necesitan para favorecer la Atención a la Diversidad del alumnado y solicitarlos a la Administración o intentar conseguir subvenciones para poder financiarlo. En el centro también sería satisfactoria remodelar los recursos TIC para poder desarrollar las tareas programadas dentro del Proyecto TIC, sobre todo para desarrollar las competencias digitales (Salmerón, Rodríguez y Gutiérrez, 2010).

Se evidencia la necesidad de formar al profesorado en los siguientes ámbitos para favorecer procesos inclusivos: Orientaciones sobre las NEAE, estrategias didácticas para desarrollar el aprendizaje cooperativo, recursos didácticos para la Atención a la Diversidad, sistemas para favorecer la accesibilidad del alumnado al currículum, técnicas de diagnóstico y evaluación a nivel inclusivo (“diagnóstico alternativo"), “actuaciones de éxito” para desarrollar comunidades de aprendizaje, estrategias para desarrollar la educación intercultural en las aulas, programas de habilidades sociales, programa de desarrollo de la inteligencia emocional, técnicas de resolución de conflictos en el aula, uso de las TIC aplicadas a las NEAE a nivel inclusivo...

\section{5.- REFERENCIAS}

Agencia Europea para el Desarrollo de la Educación Especial (2003). Necesidades educativas especiales en Europa. Publicación Monográfica. Enero de 2003.

Aguado, T. (Dir.), Malik, B., Jiménez-Frías, R., Álvarez, B., Ballesteros, B., Sánchez, M., et al. (2007). Diversidad cultural y logros de los estudiantes en educación obligatoria. Informe de Investigación (20032006) [en línea]. Disponible en http://www.uned.es/grupointer/div_cult_logros_escolares.html [Consulta: 2011, 1 de septiembre].

Araque Hontangas, N. (2009). Experiencia sobre resolución de conflictos entre culturas a través de un aula-taller de Educación Intercultural. Revista Complutense de Educación, 20(1), 99-113.

Arranz, M. L. (2008). Proceso y procedimientos de evaluación inclusivos en contextos educativos. InformeCentros. Proyecto de Investigación Educativa. Consejería de Educación de la Comunidad de Madrid.

Aubert, A., Flecha, A., García, C., Flecha, R., y Racionero, S. (2008). Aprendizaje dialógico en la Sociedad de la Información. Barcelona: Hipatia.

Badia Garganté, A., y García Tamarit, C. (2006). Incorporación de las TIC en la enseñanza y el aprendizaje basados en la elaboración colaborativa de proyectos. Revista de Universidad y Sociedad del Conocimiento, 3(2), 42-54.

Colmenero Ruiz, M. J. (2006). Análisis de las percepciones del profesorado de Educación Secundaria sobre los procesos de atención a la diversidad: Su incidencia en la formación. Revista de currículum y formación del profesorado, 10(2), 1-15.

Díaz Gómez, Á., y González Rey, F. (2005). Subjetividad: Una perspectiva histórico cultural. Conversación con el psicólogo cubano Fernando González Rey. Universitas Psychologica, 4(3), 373-383.

López Azuaga, R., Rodríguez De Cossío, M. S., Sánchez Ruiz, E. M., y Lozano, J. (2010). Estudio de caso "Diverze: una experiencia de inclusión”. Resultados investigación cualitativa Universidad de Cádiz. Jornadas Diverze, etapa 2007-2010. Asociación EQUA. Universidad de Cádiz. (paper).

Lozano González, R. M. (2008). Las TIC: Herramientas de integración. Congreso Nacional "Internet en el aula: La importancia de las TIC en las aulas”, 2008, red.es, Barcelona-Granada-Madrid-Santander. 
Ma, L. (2008). The development of academic competence among adolescents who bully and who are bullied. Dissertation Abstracts International: Section B: The Sciences and Engineering, 68, 48-66.

Marín Díaz, V. (2009). Los videojuegos como elemento de formación e integración curricular. En M. López y V. J. Llorent (coord.). La discapacidad: aspectos educativos y sociales, (pp. 177-188). Málaga: Aljibe.

Martínez, R. A., y Álvarez, L. (2005). Fracaso y abandono escolar en Educación Secundaria Obligatoria: Implicación de la familia y los centros escolares. Aula Abierta, 85, 127-146.

Moliner García, O., Sales Ciges, A., Ferrández Berruelo, R., y Traver Martí, J. (2008). Análisis de las variables que facilitan y dificultan la atención a la diversidad, según la percepción de psicopedagogos y profesorado de secundaria. Revista Iberoamericana de Educación, 45/6, 1-11.

Moliner Miravet, L., y Moliner García, O. (2007). Inclusión e Integración: ¿Qué opinión tienen los profesionales de la educación sobre ambos conceptos? I Congreso Internacional de Orientación educativa y profesional. Universitat Jaume-I, Castellón. (paper).

Muntaner Guasp, J. J. (2010). De la integración a la inclusión: un nuevo modelo educativo. En P. Arnáiz Sánchez, M. D. Hurtado y F. J. Soto (Coords.). 25 años de integración escolar en España: Tecnología e Inclusión en el ámbito educativo, laboral y comunitario (pp. 2-24). Murcia: Consejería de Educación, Formación y Empleo.

Navarrete Salvador, A. (1998). Una experiencia de aprendizaje sobre los movimientos relativos del sistema "Sol-Tierra-Luna" en el contexto de la formación inicial de maestros. Investigación en la Escuela, 35, 5-20.

Pujolàs, P. (2004): Aprender juntos alumnos diferentes. Los equipos de aprendizaje cooperativo en el aula. Barcelona: Eumo-Octaedro.

Ramos Alcázar, M.J., y Martínez López, F. J. (2011). Estrategias y metodología frente al desarrollo de una acción formativa virtual: una propuesta práctica con alumnado en Educación Secundaria. Espiral. Cuadernos del Profesorado, 4(8), 32-41. Disponible en: http://www.cepcuevasolula.es/espiral.

Rodríguez Arenas, M. J. (2011). Las TIC y el trabajo colaborativo en el aula. Hernández y otros (Coord.). Experiencias educativas en las aulas del siglo XXI: innovación con TIC (pp. 82-88). Madrid. Ariel.

Salmerón Pérez, H., Rodríguez Fernández, S., y Gutiérrez Braojos C. (2010). Metodologías que optimizan la comunicación en entornos de aprendizaje virtual. Comunicar: Revista científica iberoamericana de comunicación y educación, 34, 163-171.

Sánchez Palomino, A., y Lázaro, M. (2011). Hacia una perspectiva humanista de la Educación Especial. En Sánchez, A., C. Bernal, J. J. Carrión, J. M. Granados, R. Gutiérrez, A. Luque et al. (Eds.) (2011). Educación Especial y Mundo digital (pp. 84-101). Almería: Editorial Universidad de Almería.

Susinos Rada, T.; Rodríguez-Hoyos, C. (2011). La educación inclusiva hoy. Reconocer al otro y crear comunidad a través del diálogo y la participación. Revista Interuniversitaria de Formación del Profesorado, 25(1), 15-30.

Vygotsky, L. S. (1979). The development of higher forms of attention in childhood. Soviet Psychology. 18, 67115.

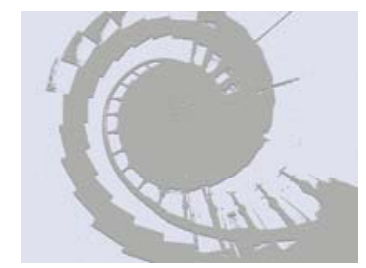

